\title{
Transatlantica
}

Revue d'études américaines. American Studies Journal

$2 \mid 2018$

Les mots pour le dire. Vocabulaire politique et propagande dans une perspective transatlantique

\section{Federal Theatre Project (1935-1939) : contexte et enjeux}

Université Toulouse - Jean Jaurès, 17-19 octobre 2019

\section{Adriana Haben}

\section{(2) OpenEdition}

\section{Journals}

Édition électronique

URL : https://journals.openedition.org/transatlantica/13540

DOI : $10.4000 /$ transatlantica. 13540

ISSN : $1765-2766$

Éditeur

Association française d'Etudes Américaines (AFEA)

Référence électronique

Adriana Haben, «Federal Theatre Project (1935-1939) : contexte et enjeux », Transatlantica [En ligne], 2 | 2018, mis en ligne le 01 février 2020, consulté le 02 février 2023. URL : http://

journals.openedition.org/transatlantica/13540 ; DOI : https://doi.org/10.4000/transatlantica.13540

Ce document a été généré automatiquement le 2 février 2023.

\section{(c) (i) (9)}

Creative Commons - Attribution - Pas d'Utilisation Commerciale - Pas de Modification 4.0 International - CC BY-NC-ND 4.0

https://creativecommons.org/licenses/by-nc-nd/4.0/ 


\section{Federal Theatre Project (1935-1939) : contexte et enjeux}

Université Toulouse - Jean Jaurès, 17-19 octobre 2019

Adriana Haben

1 Le colloque international «Federal Theatre Project (1935-1939) : contexte et enjeux », organisé par Emeline Jouve et Géraldine Prévot, s'est tenu les 17, 18 et 19 octobre à l'Université Toulouse - Jean Jaurès et au Théâtre Garonne. Cette manifestation est le fruit de la collaboration entre deux unités de recherche, Cultures Anglo-Saxonnes (CAS, EA 820, Toulouse - Jean Jaurès) et Histoire des Arts et des Représentations (HAR, EA 4414, Paris Nanterre). Durant les deux premiers jours, les interventions, sous forme d'ateliers et de conférences plénières, ont porté sur le contexte historique et sociopolitique du Federal Theatre Project (FTP), programme lancé par le gouvernement Roosevelt dans le cadre du New Deal, ainsi que sur ses répercussions, ou encore ses figures marquantes, telles que les dramaturges Hallie Flanagan et Susan Glaspell. La demi-journée du 19 octobre était quant à elle consacrée à des tables rondes autour de l'héritage du Federal Theatre Project et de la question du financement des arts vivants.

2 Le colloque a été inauguré par la conférence plénière de Jean Kempf, de l'Université Lyon 2, intitulée «Retrouver la 'promesse de la vie américaine'. Le New Deal et la culture ». Cette conférence a été l'occasion d'évoquer le contexte socio-politique du New Deal, et de passer en revue l'historiographie des années trente pour en nuancer certains aspects, en particulier la dimension inclusive des programmes du New Deal. J. Kempf a également décrit les années trente comme un tournant dans l'histoire culturelle des États-Unis, marqué par la dissolution des identités ethniques au profit de l'émergence d'une identité américaine, à travers l'intervention étatique mais aussi l'essor de la consommation. L'emphase mise par les programmes du New Deal sur les arts s'inscrit dans un contexte de révolution culturelle portée par les technologies de masse. La fin de la conférence invitait à s'interroger sur l'héritage du New Deal dans le domaine de la culture, envisagée à la fois comme création artistique et comme outil d'éducation, et non comme simple instrument de résolution de crise. La discussion qui 
a suivi a porté sur la nostalgie d'une société homogène, sans classes sociales, souvent associée au New Deal et aux années trente.

3 Le premier atelier a permis de mettre en lumière les productions culturelles des divers programmes de la Works Progress Administration (WPA) comme lieux de réflexion sociale pendant les années du New Deal, à travers des initiatives parallèles au Federal Theatre Project ou par le biais des sections locales de ces programmes.

4 Claudie Servian, de l'Université Grenoble Alpes, a ainsi présenté dans sa communication le Federal Dance Project qui a contribué à faire de la danse une arme dans la lutte des classes et un moteur d'intégration. Des origines du projet dans le « New Dance Group ", un ensemble de danseurs et de chorégraphes fondé en 1932, à sa concrétisation avec la création du Federal Dance Project, C. Servian a évoqué plusieurs de ses figures marquantes (les danseuses et chorégraphes Helen Tamiris, Anna Sokolow, Jane Dudley) et productions majeures. Le programme accueillait des artistes d'origines diverses, qui ont aidé à populariser une "danse référentielle ", profondément engagée, en dépit des critiques acerbes adressées aux danseuses en raison du caractère souvent lugubre et pessimiste des mises en scènes.

Herman Farrell, de l'Université du Kentucky, s'est intéressé dans sa communication à un moment clé de l'histoire du Federal Theatre Project: l'atelier de six semaines organisé par Hallie Flanagan lors de l'été 1937, destiné à améliorer la qualité des productions théatrales proposées. H. Farrell a ainsi retracé la genèse de ce projet, à l'issue duquel l'une des créations les plus célèbres du Federal Theatre Project, le « Living Newspaper » One-Third of a Nation, a vu le jour. L'été 1937 constitue un tournant décisif dans l'existence du Federal Theatre Project, notamment en raison de l'insistance d'Hallie Flanagan sur l'expérimentation théâtrale et son refus d'un réalisme traditionnel sur scène: les partis pris esthétiques favorisés durant ces quelques semaines ont par la suite guidé les productions ultérieures du projet.

6 Le premier atelier s'est achevé avec la communication de Nancy Jones, de l'Université du Kentucky, qui portait sur l'impact des antennes locales du Federal Theatre Project, à travers l'exemple concret de la ville de Cincinnati dans l'État de l'Ohio. Nancy Jones a ainsi noté que les divers spectacles mis en scène à Cincinnati ont mobilisé des artisans locaux pour la construction des décors ou l'élaboration des costumes, à une période où la ville était durement touchée par les effets de la Grande Dépression. Les traces de la présence du Federal Theatre Project dans la ville sont encore visibles de nos jours, à travers l'existence de nombreuses troupes de théâtre. À l'issue de ce premier atelier, la discussion s'est orientée vers les autres sections locales du Federal Theatre Project, ainsi que sur l'importance du théâtre amateur lorsqu'il s'agit d'évoquer sur scène des problématiques sociales.

7 Laura Streett, archiviste à Vassar College, dans l'État de New York, a abordé dans sa conférence plénière la vie d'Hallie Flanagan, directrice du Federal Theatre Project entre 1935 et 1939. Après un passage en revue des lieux et moments clés de la vie de Flanagan, Laura Streett a présenté son travail d'archiviste et détaillé le contenu des collections consultables à Vassar College, comprenant des photographies, certains objets personnels ainsi que des lettres. Les questions ont ensuite porté principalement sur le travail d'archiviste et l'échange de documents entre les différents fonds et institutions, ainsi que sur une éventuelle digitalisation prévue de l'archive d'Hallie Flanagan. La conférence de Laura Streett a été suivie de la lecture de la pièce Playground: The Hallie Flanagan Project par les étudiants de Dickinson College (Toulouse) 
et de l'université Toulouse - Jean Jaurès. La pièce retrace les années d'Hallie Flanagan à Vassar College. Après cette lecture, une séance de questions avec la dramaturge Mattie Brickman a permis d'évoquer son parcours professionnel ainsi que les origines de la pièce.

Le deuxième atelier, organisé par l'International Susan Glaspell Society, a confronté divers points de vue sur une autre figure emblématique du Federal Theatre Project : la dramaturge Susan Glaspell, directrice de la subdivision responsable de la région du Midwest. Dans la première communication, intitulée «The Provincetown Players and the Federal Theatre: The Essay Susan Glaspell Never Wrote ", Noelia Hernando-Real de l'Université Autonome de Madrid a détaillé l'influence des Provincetown Players, troupe à laquelle appartenaient Susan Glaspell et son mari George Cram Cook, vis-à-vis du Federal Theatre Project. Son analyse reposait principalement sur des notes et brouillons de Glaspell pour un projet d'essai sur les liens entre les Provincetown Players et le Federal Theatre Project: l'essai n'a jamais vu le jour, suite à la démission de Glaspell du Federal Theatre Project en 1938, mais selon N. Hernandez-Real, ces archives suggèrent que Glaspell voyait dans les projets d'Hallie Flanagan la continuité des idées de George Cook, décédé prématurément en 1924.

9 Linda Ben-Zvi, de l'Université de Tel Aviv, s'est également penchée sur la période durant laquelle Glaspell était à la tête du Midwest Bureau du Federal Theatre Project. Elle a centré son intervention sur un exemple marquant, le "Living Newspaper " intitulé Spirochete, traitant des maladies vénériennes et plus particulièrement de la syphilis : le titre de la pièce fait en effet référence à la bactérie à l'origine de cette infection. Le rôle marquant de Glaspell dans le conflit qui opposa Arnold Sungaard, auteur de la pièce, et les dirigeants du Federal Theatre Project en 1938 a aussi été évoqué : ces derniers refusaient alors d'accorder à Sungaard les droits d'auteurs relatifs à son œuvre, qu'il finit par obtenir après l'intervention de Glaspell.

La dernière communication de cet atelier portait sur un autre personnage important des Provincetown Players, le dramaturge et poète Alfred Kreymborg: Drew Eisenhauer, de l'Université de Strasbourg, a souligné que les contributions de Kreymborg au théâtre américain et au Federal Theatre Project ont souvent été délaissées au profit de ses travaux poétiques, davantage reconnus. Néanmoins, Kreymborg est à l'origine d'œuvres théâtrales majeures au sein du Federal Theatre Project en tant que dramaturge ou que metteur en scène, notamment America, America: a Mass Recital (1934), traitant de la misère abjecte dans laquelle vivent certaines couches de la population américaine, et The Dance of Death, pièce de W.H. Auden mise en scène par Kreymborg en 1936. Les œuvres théâtrales de Kreymborg étaient majoritairement expérimentales, et contrastaient avec le goût pour le quotidien, l'ordinaire et le vernaculaire américain qu'il cultivait en poésie.

11 À l'issue de cet atelier, les discussions se sont tournées vers les raisons de la déception de Susan Glaspell vis-à-vis du Federal Theatre Project : d'après Noelia Hernando-Real, le conflit autour des droits d'auteur de Spirochete a entrainé la démission de Glaspell, qui ne s'est pas sentie soutenue par Hallie Flanagan. Linda Ben-Zvi a également répondu à des interrogations sur l'existence d'archives prouvant l'influence de Glaspell sur l'écriture de Spirochete: selon elle, Susan Glaspell est bien à l'origine du projet, même si Sungaard demeure le seul auteur de la pièce.

12 La deuxième journée du colloque s'est ouverte sur la conférence plénière de François Thomas, de l'Université Sorbonne Nouvelle - Paris 3, intitulée «Orson Welles et ses 
compositeurs : une cohésion autant sociale et politique qu'artistique ». La conférence portait sur les quatre spectacles organisés par Orson Welles et le producteur John Houseman dans le cadre du Federal Theatre Project, entre 1936 et 1937, et sur leur choix de faire appel à plusieurs jeunes compositeurs américains, parmi lesquels Virgil Thomson, Paul Bowles, Marc Blitzstein, et Aaron Copland. F. Thomas a souligné l'attitude militante de ce groupe informel de compositeurs, et particulièrement de l'un d'entre eux, Marc Blitzstein, auteur de l' «opéra prolétarien" The Cradle Will Rock (1937). Selon François Thomas, l'engagement politique de Welles et Houseman, grandement influencés par Blizstein, a précipité leur rupture avec la WPA, suite à l'interdiction de représentation qui frappa la première de The Cradle Will Rock. La cohésion politique entre Welles, Houseman et ce groupe de compositeurs se traduit également par une cohésion esthétique : tous rejettent le romantisme allemand et sont fortement influencés par Erik Satie. F. Thomas a noté en guise de conclusion que, si aucun de ces compositeurs n'a collaboré par la suite avec Welles dans ses œuvres cinématographiques, certains de leurs choix esthétiques ont trouvé un prolongement dans les films de Welles et dans les spectacles du Mercury Theatre, groupe fondé par Welles et Houseman en 1937.

La journée s'est poursuivie avec le troisième atelier, qui comportait deux communications dans la continuité thématique de la conférence plénière : Julie VatainCorfdir (Sorbonne Université) a ainsi abordé l'adaptation du Dr Faustus de Christopher Marlowe par Orson Welles, tandis que Jeffery Kennedy (Arizona State University) a consacré sa communication à la première représentation de l'œuvre de Blitzstein, The Cradle Will Rock.

Dans sa communication intitulée « Illusions on black velvet: Orson Welles' Dr Faustus », Julie Vatain-Corfdir a analysé l'une des productions majeures de Welles et Houseman dans le cadre du Federal Theatre Project, qui se démarquait par son caractère apolitique. Le texte de Marlowe a été fortement remanié par Welles, qui n'a gardé que la moitié des répliques et les a réorganisées. La mise en scène imaginée par Welles, d'apparence simple, était en réalité extrêmement technique : les jeux d'éclairage en particulier, grâce à l'utilisation pionnière à l'époque d'un projecteur ellipsoïdal, étaient si essentiels qu'ils figuraient dans le script de la pièce. Néanmoins, malgré la qualité de la production, celle-ci a été vivement critiquée pour son absence de portée sociale ou politique.

15 L'intervention de Jeffery Kennedy portait sur la journée du 16 juin 1937, date programmée de la première représentation de l' « opéra prolétarien » de Blitzstein, The Cradle Will Rock. La première n'a pas pu avoir lieu en raison d'un décret de la WPA prononcé le jour même, qui suspendait toutes les nouvelles représentations jusqu'au $1^{\mathrm{er}}$ juillet 1937. J. Kennedy a noté que la décision de la WPA d'interrompre les représentations était probablement liée aux violentes émeutes survenues à Chicago quelques jours auparavant, et non au caractère polémique de The Crade Will Rock. Welles et Houseman ont néanmoins bravé l'interdiction de la WPA: après un changement de lieu, l'œuvre de Bliztman a finalement été jouée par ce dernier au piano, tandis que les acteurs ont chanté depuis le parterre. À l'issue de la communication de J. Kennedy, les discussions ont porté sur les récits parfois contradictoires du déroulé de la journée du 16 juin 1937 : Ilke Saal a ainsi fait remarquer que, d'après certains historiens, Welles et Houseman auraient en grande partie orchestré le chaos de cette journée pour des raisons commerciales. 

Caen Normandie, s'est penchée sur la mise en scène d'un langage dramatique de la nourriture dans la pièce de Clifford Odets, Awake and Sing (1935), dont la traduction en yiddish a été incluse dans le Federal Theatre Project. Son analyse a souligné l'importance des scènes de repas dans le contexte de la crise des années trente, ainsi que la volonté d'Odets de mettre en scène des moments de la vie ordinaire d'une famille, tout en suggérant leur résonnance avec des problématiques sociales plus larges. La communication suivante, présentée par Michael Selmon (Alma College, Michigan), portait également sur une production du Federal Theatre Project: Power (1937), pièce écrite par Arthur Arent pour relater les difficultés et conflits sous-jacents à la mise en place d'un approvisionnement électrique dans le massif montagneux des Appalaches. Après avoir souligné l'importance des réseaux qui ont permis à la pièce de voir le jour (chaque production régionale employant plus de 120 personnes), M. Selmon a évoqué l'aspect métathéâtral de la pièce, dont le script et la mise en scène attiraient sans cesse l'attention du spectateur sur l'importance de l'électricité au théâtre, pour permettre des effets de sons et de lumières. Paul-Valéry à Montpellier, qui proposait une vue d'ensemble sur les productions de Zora Neale Hurston au sein de la « Negro Unit » du Federal Theatre Project à New York. L'un des projets principaux de Hurston, une adaptation de Lysistrata, n'a ainsi jamais vu le jour sur scène. Néanmoins, Claudine Raynaud a noté que l'influence de Hurston a été capitale pour les 23 « Negro Units » disséminées à travers le pays, surtout à travers son choix de mettre en avant dans les spectacles et mises en scènes divers éléments appartenant au folklore afro-américain, comme des danses ou des chants religieux (« negro spirituals»).

18 La deuxième journée s'est poursuivie avec la conférence plénière d'Elizabeth Osborne (Florida State University), intitulée: "Has It Can't Happen Here happened? Performances of History in Times of Crisis ». L'adaptation théâtrale du roman de Sinclair Lewis a été l'un des grands succès du Federal Theatre Project, en raison de son message antifasciste : selon E. Osbourne, la pièce donnait à voir un futur potentiel sur l'espace imaginaire de la scène, dans un but à la fois didactique et préventif. La pièce a été de nouveau adaptée en 2016, peu après l'élection de Donald Trump, par le Berkeley Repertory Theatre, qui a encouragé la tenue de représentations gratuites à travers le pays. Malgré des changements substantiels par rapport à la version de 1936, E. Osborne a remarqué que la réadaptation de cette pièce s'appuyait sur un moment fort de l'histoire des États-Unis afin de le réactualiser pour le rendre à nouveau pertinent aux yeux d'un public du $\mathrm{xxI}^{\mathrm{e}}$ siècle. Les questions à l'issue de la conférence plénière ont concerné majoritairement les différences relevées par E. Osborne entre la version de 1936 et la version de 2016, ainsi que l'existence éventuelle de documents traitant des difficultés qu'aurait éprouvées Lewis à adapter son roman en pièce de théâtre. et abordait les liens entre le Federal Theatre Project et des problématiques de santé publique. Jordana Cox, de l'Université de Waterloo (Canada), est ainsi revenue sur la pièce de Sungaard, Spirochete: à partir de cet exemple, elle a analysé dans quelle mesure les « Living Newspapers » du Federal Theatre Project ont contribué à repousser les limites de l'actualité («newsworthy »). Spirochete a favorisé la mise en place d'une "poétique de l'exposition", à travers une série de stratégies destinées à rendre 
l'information publique, sur scène comme ailleurs: à l'issue des représentations, les membres du public pouvaient ainsi se faire dépister gratuitement grâce aux médecins présents bénévolement sur place.

Dorothy Chansky (Texas Tech University), présidente de l'American Theatre and Drama Society, a quant à elle présenté une autre pièce, All the Living de Hardie Albright (1938), dont l'intrigue se déroule dans un asile psychiatrique, et qui dénonce les traitements administrés aux patients et les conditions de travail des soignants. D. Chansky a passé en revue les différentes sources qui ont inspiré l'écriture de All the Living, notamment l'ouvrage de V.R. Smalls, I Knew 3000 Lunatics, ainsi que le rôle des « lecteurs » du Federal Theatre Project, chargés de relire les scripts pour sélectionner les projets, dans l'élaboration de la pièce.

21 La communication de Kate Dossett, de l'Université de Leeds, abordait la question de la santé sous un angle métaphorique, à travers la problématique de la «santé » des archives. Elle a présenté ses travaux sur les manuscrits d'auteurs noirs dans les archives de la Bibliothèque du Congrès, soutenant que ces documents, qui n'ont jamais été portés sur scène pour la plupart, sont des ressources tout aussi précieuses dans l'élaboration de l'histoire du Federal Theatre Project que les manuscrits officiels ou publiés. Selon elle, la méfiance est de rigueur face à des histoires du Federal Theatre Project constituées uniquement à partir de manuscrits publiés, car celles-ci ne représentent que le point de vue des blancs, qui étaient très souvent les seuls décideurs des projets qui voyaient le jour.

Une dernière conférence plénière, donnée par Ilka Saal (Université d'Erfurt), est venue clore la deuxième journée du colloque. Intitulée « "As American as Walt Disney": The Political Theatre of the Living Newspaper ", elle portait sur le format théâtral du "Living Newspaper ", fortement inspiré par les gros titres de l'actualité et destiné à une audience très large. Le succès de ce format est dû, selon I. Saal, à un heureux mélange entre l'esthétique du théâtre politique (empruntée à Brecht et Piscator) et des techniques de divertissement bourgeois. Les «Living Newspapers» étaient «aussi Américains que Walt Disney", d'après la formule d'Hallie Flanagan, car ils s'intéressaient principalement aux problèmes rencontrés par le citoyen lambda, et critiquaient les excès du système capitaliste tout en proposant des solutions à l'intérieur même des paramètres de ce système. Suite à la conférence d'I. Saal, les derniers échanges se sont orientés sur l'héritage du Federal Theatre Project : certains ont ainsi estimé que les «Living Newspapers» constituaient un véritable outil de changement, tandis que d'autres intervenants ont évoqué la pièce d'Arthur Miller, Death of a Salesman, comme héritière de l'ethos du FTP, dans sa manière d'aborder des questions politiques à travers la sphère du personnel.

23 La dernière demi-journée du colloque, qui s'est déroulée le 19 octobre au Théâtre Garonne, était organisée sous la forme de tables-rondes autour de la question du financement des arts vivants: le FTP étant une politique de financement des arts exceptionnelle dans l'histoire des États-Unis, il s'agissait de s'interroger sur l'héritage d'un tel programme et sur l'existence éventuelle de dispositifs similaires dans d'autres pays. Lors de la première table ronde, Stéphane Gil, directeur délégué du Théâtre de la Cité, a souligné l'existence en France de financements publics assurés par les collectivités territoriales, tout en notant que ceux-ci étaient parfois difficiles à obtenir selon la localisation géographique. Rima Abdul-Malak, ancienne attachée culturelle à l'ambassade de France aux États-Unis, a quant à elle rappelé le caractère hors norme de 
la politique du Federal Theatre Project dans un pays où la seule agence gouvernementale de financement des arts, le National Endowment for the Arts, représente seulement $0,04 \%$ du budget fédéral. Le financement des arts vivants aux États-Unis relève ainsi majoritairement de la philanthropie ou du mécénat. Enfin, la dernière intervenante, Harriet O'Malley, attachée culturelle australienne à l'ambassade de Paris, a noté qu'il existe en Australie des dotations prévues pour les projets destinés à contribuer au rayonnement du pays à l'international. Néanmoins, les financements de l'Australia Council for the Arts sont principalement réservés à la " grande culture » ou à la reconnaissance des arts aborigènes, et il est plus difficile pour les projets indépendants ou les artistes émergents d'en obtenir.

La deuxième table ronde réunissait divers intervenants autour de la question des financements des arts en temps de crise. Emmanuel Wallon et christian Biet (professeurs respectivement de sociologie et de théâtre à l'Université de Nanterre) ont ainsi discuté d'une éventuelle " américanisation » des systèmes de financement, par le biais d'un recours accru aux recettes annexes et au mécénat, une idée soulignée par l'évocation de modèles hybrides de financement dans l'intervention de Stéphane Boitel, co-programmateur au Théâtre Garonne. Eric Fourreau, directeur des éditions de l'Attribut, a quant à lui abordé la question de la légitimité de la culture et de son financement: la culture doit-elle être soumise à une logique de rentabilité ? La discussion s'est ensuite orientée vers le modèle américain, Rima Abdul-Malak et Harriet O’Malley remarquant que la « loi du marché » américaine a paradoxalement contribué à créer davantage de diversité dans la représentation des minorités raciales et de genre, sur scène comme dans les salles de théâtre.

Enfin, la manifestation s'est clôturée sur une dernière table ronde, laissant la parole aux artistes sur la question de l'impact des politiques de financement sur leur travail de création. Deux artistes australiens, Leah Shelton (de la compagnie Terror Australis) et Adriano Cortese (de la compagnie Ranters Theatre), ont ainsi décrit les difficultés traversées par les artistes au quotidien pour obtenir des subventions et parvenir à vivre de leur art. Les dernières discussions avec Alison Halit, productrice et curatrice, ont illustré l'influence que peuvent avoir les organismes de financement sur la nature, la nomenclature, le contenu ou encore le déroulement d'un spectacle : en Australie, par exemple, les artistes doivent désormais rendre des comptes aux organismes financeurs sous forme de rapports à effectuer après l'aboutissement du projet.

Le colloque a ainsi permis d'envisager le Federal Theatre Project comme une période exceptionnelle dans l'histoire des États-Unis en matière de financement des arts vivants et d'implication de l'État dans la culture. Au cours des trois journées, les points de vue des chercheurs se sont confrontés à ceux des artistes et d'autres acteurs culturels afin de proposer des approches complémentaires de ce projet, dont l'héritage et les répercussions ont suscité de vives discussions. 
INDEX

Thèmes : Actualité de la recherche

\section{AUTEUR}

ADRIANA HABEN

Université Toulouse - Jean Jaurès 